\title{
USING P2P NETWORKS FOR ERROR RECOVERY IN MBMS APPLICATIONS
}

\author{
Praveen Sanigepalli ${ }^{\dagger}$, Hari Kalvat, and Borko Furht \\ ${ }^{\dagger}$ Motorola, Plantation, FL \\ ${ }^{\ddagger}$ Dept. of Computer Science and Engineering, Florida Atlantic University, Boca Raton, FL 33431
}

\begin{abstract}
The wireless networks are notoriously error prone and all errors cannot be prevented in real-time communications. The problem of error correction becomes even more challenging in mobile multicast/broadcast applications. The mobile devices are being equipped with multiple modems that could work simultaneously; for example, devices with both GSM (WAN) and WLAN networks such as WiFi. These multi-modal devices can use the second network to improve their error resilience. We propose a P2P approach to establish and utilize an error recovery channel on a secondary network for multi-user video applications. The mobile devices within the vicinity can utilize the WLAN network to form a P2P network for error recovery purpose. We developed and evaluated three error recovery models for error recovery over secondary networks. The proposed models balance the response time, bandwidth utilization, fairness, and unnecessary data received.
\end{abstract}

\section{INTRODUCTION}

Multimedia broadcast services on mobile devices are becoming popular for broadcasting small clips of video to even receiving number of television channels. Video multicasting is seeing renewed interest from mobile service providers where spectrum is scarce and multicasting allows a larger user base. 3GPP is currently working on a standard for Multimedia Broadcast and Multicast Services (MBMS) [3]. Streaming of live or stored video content to group of mobile devices comes under the scope of MBMS. Some of the typical applications are subscription to sports, music videos, news clips, weather information and live TV content.

MBMS specifies transmission of data packets from single entity to multiple recipients using a common broadcast channel. This is much more efficient than IP multicast where packets are duplicated for each recipient in a broadcast/ multicast group. The summary of Radio Access Network (RAN) requirements is presented in [1]. The video error resilience in such services is critical to maintain consistent broadcast quality for terminals.
The mobile handsets experience wide variety of network conditions such as fading, diversity of signal strength conditions and interference. The transmission of video over wireless channels is very challenging especially with realtime requirements in a session shared by multiple users. The members of the multicast or broadcast session are likely to experience diversity of network conditions with varying device capabilities; maintaining video quality of a multicast session by considering the needs of all participants is a challenging problem and requires significant research in this area. The traditional error resilience techniques for multicasting focused on FEC or retransmission and are applied to the Internet or ATM systems [2,3]. However, MBMS presents different paradigm due to mobility, diversity of signal conditions, low power and spectrum utilization requirements of receivers and requires new approaches to error resilience.

The next generation mobile devices are being equipped with multiple modems that could work simultaneously. An example would be a Motorola Seamless mobility device that can work on both GSM and WLAN networks. We exploit this multiple modem capability to provide significantly higher video quality to mobile receivers through lost packet recovery over secondary networks. With this approach, for example, a video session is broadcast/multicast over GSM networks while a secondary network, formed among a set of receivers using $802.11 \mathrm{a} / \mathrm{b}$ connectivity, is used for error recovery without a need for server based error recovery. The main contributions of this paper are the design and analysis of lost packet recovery schemes over peer-to-peer secondary networks that improve the quality of broadcast/multicast video.

The paper is organized as follows: the MBMS architecture and concepts are introduced in section 2, the proposed peer-to-peer error resilience schemes are presented in section 3, section 4 presents the experimental results, and the conclusions are presented in section 5 .

\section{MBMS ARCHITECTURE}

MBMS standards enable multimedia broadcast, multicast, and asynchronous download services to mobile users within a cell. The goal of the MBMS standardization activity is to 
standardize components and interfaces in the system architecture that would eliminate ambiguity and promote synergy between network operators, content providers, handset manufacturers and network manufacturers.

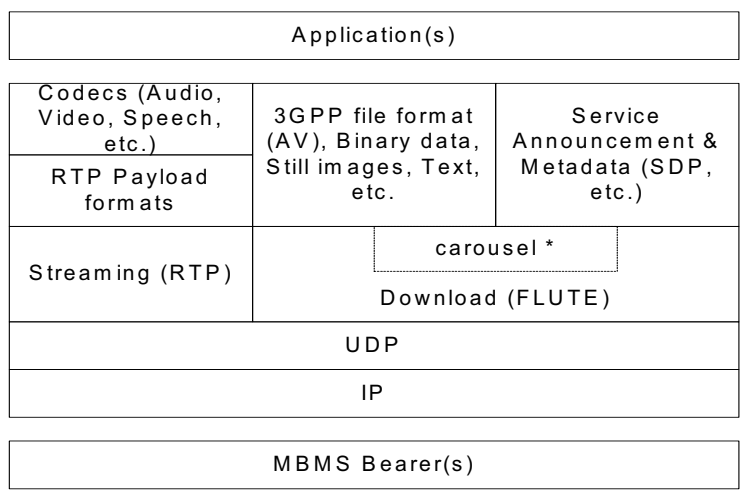

Figure 1 Protocols for MBMS Streaming

MBMS User Service Discovery/ Announcement are required for advertising MBMS Streaming in advance of, and potentially during, the User Service sessions. They involve delivery of fragments of metadata to many receivers in a suitable manner. The metadata itself describes the details of the services. A metadata fragment is a single uniquely identifiable block of metadata and could be described as an SDP file. The metadata consists of description of details about MBMS user services, MBMS user service sessions, associated delivery methods and service protection. The session description protocol (SDP) is also used to describe the multimedia delivery session. The Real-time Transport Protocol (RTP) is used for delivery whereas RTCP is used for statistics on channel quality. The RTP and RTCP protocols are delivered over UDP/IP protocols. The MBMS bearer services are used to deliver over the MAC and physical layers.

\section{ERROR RECOVERY OVER P2P NETWORKS}

More and more mobile devices are being equipped with multiple modems that could work simultaneously. Typical WLAN networks that devices are equipped with are Bluetooth and $802.11 \mathrm{a} / \mathrm{b}$ Wireless LAN (WLAN). These local area networks offer higher throughput; however, the range of communication is limited. The spectrum used in such networks is unlicensed so that bandwidth can be utilized by the mobiles without any cost. Since bandwidth is a premium resource and contentious on WAN, the mobile devices can utilize the WLAN network to form an adhoc network if they are within the vicinity. The members of the P2P network can cooperate to perform error recover during a multicast/broadcast session. The error recovery using the WAN is not desirable, since the loss could be caused by congestion and additional transmissions could aggravate the congestion. Lost packet recovery through packet retransmission and selective retransmission has been studied in the past [4]. Prior work on error resilience in IP multicast has focused on FEC based techniques and secondary channels have not been explored [2,5]. In this paper we present the design and analysis of novel error resilience techniques where an adhoc network on WLAN is used to recover errors. The error resilience techniques vary based on the application requirements and network characteristics. For broadcast or multicast applications, the error resilience techniques should be designed to scale appropriately based on the number of users. This would limit the applicability of error resilient techniques such as retransmissions of lost packets. The interactive techniques that require close interaction between encoder and the decoder are also not appropriate for multi-user applications. Figure 2 depicts the concept of error resilient network formation.

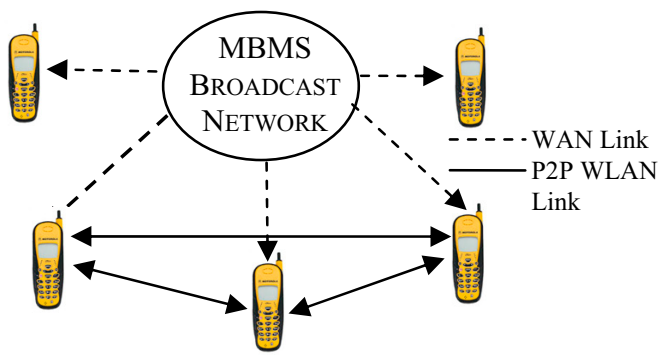

Figure 2. P2P error resilience network in MBMS

The provisioning of reliable multicast services is a challenging problem. The key characteristics of IP-based multicast services on wired networks are listed below [2]:

- Multiple receivers with heterogeneous connections and processing capabilities

- Feedback implosion: if every receiver transmits feedback on the quality of reception, the sender will be overwhelmed.

- For a multicast tree with a common link and individual links to all receivers, loss on a common link results in many receivers losing the same packet.

In MBMS environment, all the users are connected over a common bearer but can suffer different losses because of diverse conditions at the receiver. Because of this the likelihood of all the users loosing the same packet is very small. With at least one user highly likely to have received the correct packet, P2P approaches to error recovery can be highly effective. To accomplish such packet recovery, three schemes are proposed and are compared using metrics such as bandwidth requirements, overall delay, fairness, and lost packets. The first scheme uses a centralized scheme with a leader that plays a pivotal role in packet recovery. The transport in this scheme uses unicast so it is noted as unicast scheme. In the second scheme, a distributed scheme is proposed where the packets are multicast and each receiver plays an equal role in packet recovery. In the third scheme, 
the leader role still exists, but the recovery is handled by all members. This would share the transmit burden among the users for the session. In these schemes, broadcast network is referred to as Wide Area Network (WAN) whereas local area network is referred to as WLAN. MBMS streaming services use IP-based RTP/UDP protocols for transport of video packets over the network. The RTP headers consist of sequence numbers to be used for detecting packets coming out of order. Also using such sequence numbers, packet loss can be detected. Upon detection of a loss and assuming an adhoc channel has been formed, the proposed error recovery schemes can be utilized.

\subsection{Point Coordinated Error Resilience Channel}

In the Point Coordinated Channel (PCC), a designated leader is at the center of the packet recovery operations. In this scheme, for each packet broadcasted on WAN, the members of group determine if they have incurred a loss. This loss is indicated to the leader in the form of a NAK packet. A positive ACK from each group member to the leader is not preferred; as in a large group, these ACKs could overwhelm the leader. The leader assumes if a NAK is not received, then the member has received the packet correctly. If the leader has received the WAN packet correctly, it would unicast the packet to the receivers that have sent a NAK. If the leader does not have the packet, it would receive the packet from a receiver randomly picked from the successful receivers and then unicast the packet to the receivers that have sent a NAK. Typically, leader-based schemes impose a significant load on the leader in terms of processing power and battery life. This is not desirable for a typical mobile. However, the leader role can be played by a host, such as a laptop or a server that does not have power constraints like a mobile.

\subsection{Distributed Error Resilience Channel}

The unicast based schemes do not scale well with large groups. As group size and loss rates increase, the bandwidth requirements and the delay will also increase proportional to loss rates. A multicasting scheme involves sending the same copy to all the users regardless of their link conditions. For each packet that is multicast, there are no retransmissions at the link layer (e.g., WiFi multicast) to ensure stability and avoid NAK explosion. However, an application-level retransmission can be applied to protect against losses. Typically, applications apply extra FEC to the data packets. If the link layer passes damaged packets to the application, the errors could be corrected. This scheme uses a decentralized leadership where receivers responsible for retransmitting packets can change for each packet. A receiver that has lost a WAN packet would multicast a NAK on the adhoc channel. The other receivers that also lost the packets would not transmit a NAK upon receiving the first receivers NAK. This is also referred to as a NAK suppression scheme. Upon receiving a NAK, a mobile that has received the packet correctly would multicast transmission request packet. Similar to NAK suppression, the other receivers that received the packet correctly, would suppress their transmission requests. The mobile that sends first transmission request would multicast the video packet to the group thereby resulting in packet recovery for all the receivers with that lost packet.

\subsection{Fair Load Point Coordinated Error Resilience Channel}

In this mechanism, a hybrid between a leader-based scheme and a distributed scheme is implemented that is fair in terms of transmission load at the same time providing a reliable transmission. In Unicast scheme (PCC), the leader is burdened with transmission to each unsuccessful receiver which drains the leader's resources. A modification of the unicast scheme called Fair Load Point Coordination Error Resilience Channel (FPCC) rotates the transmission duties to a randomly picked successful receiver. The leader receives the unicast NAK packets from individual receivers and determines the status of every packet at each receiver. Among the successful receivers, it would randomly select one for the retransmission role for a specific packet. This designated transmitter would then multicast the packet to all users. This scheme can balance the transmission burden on each receiver. As packet transmissions consume significant power, transmission load balancing is highly desirable.

\section{RESULTS AND DISCUSSION}

Simulations were done using a combination of Opnet and Visual $\mathrm{C}++$ model for performance evaluation. The available Opnet models have a coarse model of the physical layer losses and cannot be modeled accurately for wireless channels. Using Opnet, certain characteristics of the WLAN MAC layer, such as packet transmission delays, are determined. The outcomes such as average delay for WLAN adhoc network are fed into a Visual $\mathrm{C}++$ model that simulated a broadcasting scheme in secondary networks. Since several schemes are proposed for packet recovery, the following key metrics are used to characterize the performance: 1) WLAN bandwidth utilization 2) maximum delay for packet recovery 3) average delay for packet recovery 4) number of unrecoverable packets (even if one mobile cannot recover a packet, it is assumed that the group has lost the packet) 5) transmitted bandwidth per member 6) total number of packets transmitted per member. 7) Unnecessary received bandwidth by group member. The total transmitted packets and the unnecessary packets received have an impact on battery life as packet transmission on a WLAN consumes twice the power required to receive a packet and affects battery life. 
Excessive transmissions and unnecessary receptions are not desirable for a mobile. Because of space limitations, only some of the results are discussed in this paper.

Experiments were conducted where the above seven metrics were calculated for the predetermined simulation duration of 30 seconds of video multicasting. The video sequence assumed for the transmission is of QCIF resolution coded at $128 \mathrm{kbps}$ and a frame rate of $15 \mathrm{FPS}$. The transmission of video sequence is simulated with packet length of 1500 bytes for I packets and 250 bytes packet for $P$ packets. Assuming WLAN segmentation threshold at 250 bytes, the WLAN transmitting packet size was kept constant for either P or I packets. For each of these experiments, several trials are conducted and their average is reported.

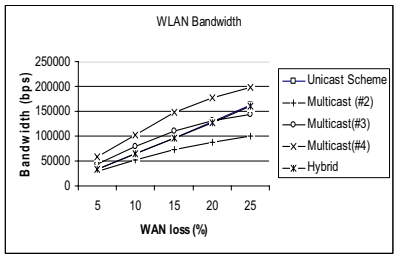

(a)

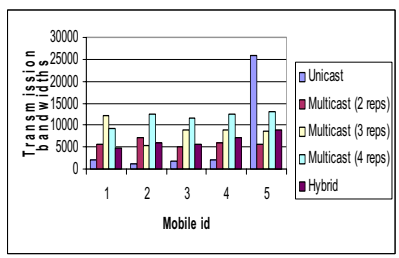

(c)

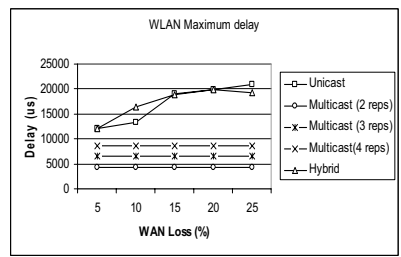

(b)

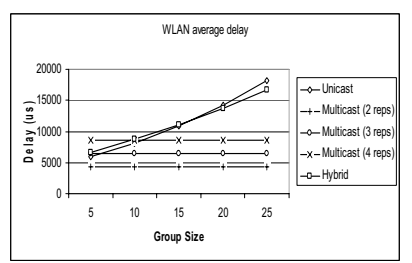

(d)
Figure 3. Simulation Results

Figure 3.a shows the impact of WAN losses on the retransmission bandwidth on the WLAN with the group size and WLAN losses are kept constant. The group size is 5, the WLAN loss is constant for all group members at 5\%, whereas the WAN loss is varied from $5 \%$ to $25 \%$. The simulations for multicast scheme are also conducted with each WLAN packet being repeated 2, 3 or 4 times to increase the reliability of multicast packet transmissions. Each experiment is repeated for three times and the averaged results are reported for each metric. For all the schemes, as WAN loss rates increase, the bandwidth requirements also increase. In the Figure 3.b shown are the maximum delays experienced by the group. In the figure, again the maximum delay increases with WAN loss in unicast/hybrid schemes whereas in the multicast scheme the maximum delay remains constant. As packet repetitions increase in multicast scheme, the average delay increases; however, it stays constant independent of WAN loss rate. Also, the maximum delay experienced is worse in unicast and hybrid schemes compared to the multicast schemes.

The individual mobile transmission bandwidths are shown in Figure 3.c. With this metric, it can be determined that the unicast scheme has disproportionate transmission requirements for mobiles. Most of the transmissions are done by mobile 5 and this scheme can be utilized in use cases when a gateway or "powerful" peer (Ex: laptop) is part of the network. However, in the case of mobile devices such heavy transmission, load would take a toll on battery life. The Hybrid scheme is advantageous in such scenarios, since the error recovery transmissions are shared across group members by a leader. For this scheme, the simulations show that bandwidth transmission requirements of the mobiles are with in the same range.

Figure 3.d shows the impact of group size on average delay for packet recovery. The average delay increases with the group size. As the group size increases, the total number of WAN packet losses by mobiles also increases. In unicast/hybrid cases, as each mobile is served individually, the average delay for complete packet recovery increases linearly with group size. In the case of multicasting schemes, the average delay remains constant.

\section{CONCLUSION}

The emerging MBMS applications on mobile networks enable new class of services. The nature of MBMS, however, requires new approaches to error resilient content delivery. We exploit the multi-modal capability of devices to provide error resilient streaming services. The paper proposes a P2P error recovery mechanism for MBMS services. The proposed methods were evaluated based a set of metrics that can serve as guides in selecting the appropriate error resilience method. The power vs recovery delay tradeoffs can be addressed using the proposed schemes. The delay is an important factor as it affects the amount of buffering and startup delay at the receiver. While the simulation results are shown for video streaming, the proposed approach is applicable to other streaming media such as audio.

\section{REFERENCES}

[1] 3rd Generation Partnership Project: Technical Specification Group Radio Access Network, Multimedia Broadcast Multicast Service (MBMS), UTRAN / GERAN requirements, 3GPP TR 25.992, V6.0.0.

[2] Carle, George and Ernst W. Biersack, "Survey of Error recovery techniques for IP based audio visual multicast applications", IEEE Network, vol. 11, no. 6 (Dec. 1997):24-36.

[3] Tan, Wai-Tian and A. Zakhor, "Video multicast using layered FEC and scalable compression", IEEE Transactions on Circuits and Systems for Video Technology, vol. 11, issue 3 (March 2001):373 - 386

[4] M. Piecuch, K. French, G. Oprica and M. Claypool, "A Selective Retransmission Protocol for Multimedia on the Internet," Proceedings of SPIE Multimedia Systems and Applications, Boston, MA, USA, November 5-8, 2000.

[5] Ge, Peng and Philip K. McKinley, "Comparisons of Error control techniques for Wireless Video Multicasting", IEEE International Conference on Performance, Computing, and Communications, (2002):93-102. 